\title{
Reply: Surveillance of Crimean-Congo Haemorrhagic Fever in Pakistan
}

\author{
Ehsan Mostafavi ${ }^{*}$, Kourosh Holakouie-Naieni², Mostafa Salehi Vaziri ${ }^{3}$ \\ ${ }^{1}$ Department of Epidemiology and Biostatistics, Research Centre for Emerging and Reemerging Infectious Diseases, \\ Pasteur Institute of Iran, Tehran, Iran; ${ }^{2}$ Department of Epidemiology and Biostatistics, School of Public Health, \\ Tehran University of Medical Science, Tehran, Iran; ${ }^{3}$ Department of Arboviruses and Viral Hemorrhagic Fevers, \\ Research Centre for Emerging and Reemerging Infectious Diseases, Pasteur Institute of Iran, Tehran, Iran
}

Received Feb 05, 2018; Accepted Feb 07, 2018

To the editor: In a recent publication entitle "Surveillance of Crimean-Congo haemorrhagic fever in Pakistan" published in "The Lancet Infectious Diseases", the authors claimed that before the Eid al-Adha, CrimeanCongo Hemorrhagic Fever (CCHF)-livestock infected are imported from Iran and Afghanistan to Baluchistan province of Pakistan and then transferred to other areas of the country [1]. They claimed that the high prevalence of CCHF in Baluchistan province was merely due to the imported livestock. In a commentary, Mallhi and colleagues (2017) criticized the above mentioned claims by pointing to other factors that are more probably responsible for the high prevalence of CCHF in Baluchistan province of Pakistan [2]. Importantly, they noted that animal husbandry was the primary occupation of the majority of rural and nomadic people living in Baluchistan. This fact besides people's lack of knowledge about tick-borne diseases, proper prevention methods, unavailability of proper health-care facilities, low quality of patient care and treatment, climate change, and dry weather were highlighted as major contributing factors. The authors also considered importation of infected livestock, from Iran and Afghanistan, as a contributing factor to the CCHF epidemics in Baluchistan.

There are many flaws in the claims of Karim and colleagues (2017). There are many papers on the situation of CCHF in Pakistan [3-6]. However, no previous report on the importation of CCHF-infected livestock from Iran to Pakistan is available. Molecular epidemiology studies indicated that the CCHF viruses in Pakistan and Afghanistan were genetically similar [5], and the source of CCHF virus circulating in the Middle East was from Pakistan [7]. Moreover, some significant methodological flaws are evident in the study of Karim and colleagues (2017); their small sample size comprised only 21 blood samples from livestock that were allegedly from Iran and Afghanistan, which were not reported separately for each country. They have also neglected the infection rate in native livestock in comparison to the imported livestock. It is noteworthy that meat and livestock in Iran are more expensive than in Pakistan and Afghanistan, and hence, there is more chance of livestock influx from Afghanistan and Pakistan towards Iran $[8,9]$. This fact alone rejects the central assumption in their study on the importation of infected livestock from Iran to Pakistan. Finally, the paper does not mention the sensitivity and specificity of the ELISA assay used in the study. Some serologic tests may be non-specific to CCHF, which create the possibility of cross-reaction to other naïve viruses, like Hazara [10].

Moreover, studies on genetic diversity of CCHF in Iran and Pakistan reveals more weakness in the conclusion of Karim and colleagues (2017). Based on their claim, the circulating genotypes in Iran should also occur in Pakistan with a high degree of genetic similarity. However, out of the six identified CCHF virus genotypes, only the genotypes I, IV, V, and VI have been detected in Iran [11, 12]. Geographically, genotypes I, V, and VI are found in northern and western areas of Iran, while the circulating strains in southeastern Iran, in the proximity of Pakistan, belong to the genotype IV, i.e., the Asian genotype [13, 14]. On the other hand, all the identified strains in Pakistan are from the genotype IV, and there is no report on the other prevalent Iranian genotypes, I, IV, and V from Pakistan.

Finally, the study reports the mortality rate of $41 \%$ in Pakistan, compared to $15 \%$ in Iran [15]. This could be due to Iran's better health care quality and stronger infrastructures for identification and treatment of CCHF cases, compared to Pakistan.

Although the existing evidence suggests dissemination of the CCHF virus from Pakistan and Afghanistan to Iran, we believe that further molecular epidemiological studies are needed to shed light on this issue.

\section{ACKNOWLEDGEMENT}

We acknowledge the scientific contribution of Center for communicable disease control of Ministry of Health and the Iranian veterinary organization for supporting this paper.

*Correspondence: Ehsan Mostafavi

Department of Epidemiology and Biostatistics, Research Centre for Emerging and Reemerging Infectious Diseases, Pasteur Institute of Iran, No. 69, Pasteur Ave, Tehran, Iran, 1316943551.

Email: mostafavi@pasteur.ac.ir

Tel/Fax: +98 (21) 66496448 


\section{CONFLICT OF INTEREST}

The authors declare that there are no conflicts of interest associated with this manuscript.

\section{REFERENCES}

1. Karim AM, Hussain I, Lee JH, Park KS, Lee SH. Surveillance of Crimean-Congo haemorrhagic fever in Pakistan. Lancet Infect Dis. 2017; 17 (4): 367-8.

2. Mallhi TH, Khan YH, Tanveer N, Khan AH, Qadir MI. Commentary: Surveillance of Crimean-Congo Haemorrhagic Fever in Pakistan. Front Public Health. 2017; 5: 132.

3. Haider S, Hassali MA, Iqbal Q, Anwer M, Saleem F. Crimean-Congo haemorrhagic fever in Pakistan. Lancet Infect Dis. 2016; 16 (12): 1333.

4. Hasan Z, Atkinson B, Jamil B, Samreen A, Altaf L, Hewson R. Diagnostic testing for hemorrhagic fevers in Pakistan: 20072013. Am J Trop Med Hyg. 2014; 91 (6): 1243-6.

5. Khurshid A, Hassan M, Alam MM, Aamir UB, Rehman L, Sharif S, et al. CCHF virus variants in Pakistan and Afghanistan: Emerging diversity and epidemiology. J Clin Virol. 2015; 67: 25-30.

6. Rai MA, Khanani MR, Warraich HJ, Hayat A, Ali SH. Crimean-Congo hemorrhagic fever in Pakistan. J Med Virol. 2008; 80 (6): 1004-6.

7. Mild M, Simon M, Albert J, Mirazimi A. Towards an understanding of the migration of Crimean-Congo hemorrhagic fever virus. J Gen Virol. 2010; 91 (1): 199-207.
8. Afzal M, Ahmad S, Baloch AS, Ahmad QB. Seasonal price variation and price characteristics for small ruminants marketing in Balochistan. Pakistan J Agric Res. 2011; 24.

9. Ayyub RM, Rana A, Bagi ARA, Al-Thomaly AA. Exploring future markets for Pakistani Halal meat export. International Journal of Social Entrepreneurship and Innovation 2. 2013; 2 (1): 11-20.

10. Zeller H. Laboratory diagnosis of Crimean-Congo hemorrhagic fever. Crimean-Congo Hemorrhagic Fever: Springer; 2007: 233-43.

11. Bente DA, Forrester NL, Watts DM, McAuley AJ, Whitehouse CA, Bray M. Crimean-Congo hemorrhagic fever: History, epidemiology, pathogenesis, clinical syndrome and genetic diversity. Antivir Res. 2013; 100 (1): 159-89.

12. Salehi-Vaziri M, Baniasadi V, Jalali T, Mirghiasi SM, AzadManjiri S, Zarandi R, et al. The First Fatal Case of CrimeanCongo Hemorrhagic Fever Caused by the AP92-Like Strain of the Crimean-Congo Hemorrhagic Fever Virus. Jpn J Infect Dis. 2016; 69 (4): 344-6.

13. Chinikar S, Bouzari S, Shokrgozar MA, Mostafavi E, Jalali $\mathrm{T}$, Khakifirouz S, et al. Genetic Diversity of Crimean Congo Hemorrhagic Fever Virus Strains from Iran. J Arthropod Borne Dis. 2014.

14. Chinikar S, Shah-Hosseini N, Bouzari S, Jalali T, Shokrgozar MA, Mostafavi E. New circulating genomic variant of Crimean-Congo hemorrhagic fever virus in Iran. Arch Virol. 2013; 158 (5): 1085-8.

15. Mostafavi E, Haghdoost A, Khakifirouz S, Chinikar S. Spatial Analysis of Crimean Congo Hemorrhagic Fever in Iran. Am J Trop Med Hyg. 2013; 89 (6): 1135-41. 